\title{
Eliminating Cyanoglucosides from Cassava Tubers: Exploiting the Endogenous Turnover and Transporters
}

\author{
Samson Ugwuanyi ${ }^{1}{ }^{*}$, Chika C. Nwadinigwe ${ }^{2}$, Chidiebere A. Adeosun ${ }^{3}$ and Hannah $\mathrm{I}^{4}$, Iwuoha $^{5}$ \\ ${ }^{1,5}$ Department of Plant Breeding, Justus Liebig University, Giessen, Germany \\ ${ }^{2,3,4}$ Department of Plant Science and Biotechnology, University of Nigeria, Nsukka, Nigeria \\ Corresponding author*
}

\begin{abstract}
Cassava (Manihot esculenta Crantz) is a major staple food in sub-Saharan Africa. Its efficient utilization for food is affected by the presence of cyanoglucosides in the tubers. Following the mapping of CYP79D1/D2 genes involved in the cyanoglucosides biosynthesis, the last two decades have witnessed intense research to develop cassava plants with insignificant level of cyanoglucosides via gene silencing. These concerted efforts are yet to produce satisfactory outcomes. However, research has revealed roles of cyanoglucosides in important metabolic pathways in cassava. Therefore, an alternative direction must be sought to conserve the functions of these metabolites while eliminating the compounds from the edible tubers. The endogenous turnover and transport pathways of cyanoglucosides could be interesting areas for future research. It is expected that the upregulation of the genes involved in the turnover pathway would result in increased synthesis of these turnover products, which would subsequently be incorporated into primary metabolism; thereby avoiding the accumulation of cyanoglucosides in the tubers. Similarly, the characterization of glucosinolate transporters in Arabidopsis presents an avenue to study the mechanisms and roles of transporters of defense compounds such as cyanoglucosides in cassava. Research has shown that the metabolic engineering of these compounds are possible by interfering with the expression of transport molecules, and holds the potential for developing plants with reduced accumulation of toxic compounds in the edible tissues or plant parts. The objective of this study was to document recent literature and highlight pathways that involve turning cyanoglucosides into useful intermediates which could be exploited in developing cyanoglucosides-free cassava tubers.
\end{abstract}

Keywords: Cyanogenic glucosides, RNA interference, endogenous turnover

\section{INTRODUCTION}

$\mathrm{C}$ assava (Manihot esculenta Crantz) is a major staple in the developing countries, ranked $4^{\text {th }}$ most important crop behind maize, rice and sugarcane [1]. Cassava is an important food crop to a large part of the population in the sub-Saharan Africa and it is primarily cultivated for its tuberous roots, which provide more than half of the daily calorie intake in sub-Saharan Africa where more than $80 \%$ of the harvest is consumed [2,3]. Unfortunately, the presence of a significant amount of cyanogenic glucosides (CNglcs) in all parts of the cassava plant is the major limitation to its efficient use for food across the world [4]. In the last two decades, eliminating $\mathrm{CNglcs}$ has been a major research priority in cassava breeding programmes. Andersen et al. [4] mapped two cytochrome P450 genes: CYP79D1 and CYP79D2 involved in the CNgles biosynthesis, triggered interests in the application of different molecular techniques to interfere with the expression of these genes. Several reports have been published but there is still more work to be done in this area. However, findings from several of these researches have added to the evidence of the roles played by $\mathrm{CNglcs}$ in important metabolic pathways in cassava, further compounding this challenge. The objective of this study was to elucidate aspects of CNglcs metabolism in cassava and summarize recent research findings including the challenges encountered in this regard.

\section{CYANOGENIC GLUCOSIDES IN CASSAVA}

Cyanogenic glucosides are secondary metabolites produced by thousands of plant species found in both vascular and nonvascular plants [5]. Cyanogenic glucosides play vital roles in plant defense against herbivores and theft $[6,5]$. Aside from defensive actions, CNglcs may have a variety of additional activities in the plants, which include possible functions as reactive oxygen species (ROS) scavengers and acting as storage compounds for reduced nitrogen and carbon $[7,8,9,5,3]$. However, there are numerous more roles of cyanoglucosides in plants that are unknown.

\section{BIOSYNTHETIC PATHWAY AND CYANOGENESIS IN CASSAVA}

The detailed study of the biosynthetic pathway was first carried out in Sorghum bicolor (L. Moench) [5]. Dhurrin, which is the cyanogenic glucoside present in Sorghum, is derived from the amino acid L-tyrosine by the sequential action of two Cytochrome P450 genes, CYP79A1 and CYP71E1, to produce $\alpha$-hydroxynitrile [10]. This hydroxynitrile is subsequently glucosylated by a UDPglucose: p-hydroxymandelonitrile-O-glucosyltransferase UGT85B1 [11,12]. This basic cyanoglucoside biosynthetic pathway was presumed to be similar across many plant species, and led to the belief that a cytochrome P450 closely related to sorghum CYP71E1, is involved in the conversion of 
2-methylpropanol oxime to acetone cyanohydrin in cassava $[13,14]$.

This pathway in cassava has long been elucidated by Andersen et al. [4]. The biosynthesis of CNglcs (linamarin and lotaustralin) in cassava starts with the conversion of valine and isoleucine by cytochrome P450 enzymes, which are translation products of two genes: CYP79D1 and CYP79D2 [4]. The translation products of CYP79D1 and
CYP79D2 are similar, and catalyze the conversion of either valine, which is a high affinity substrate or isoleucine, a low affinity substrate, to their corresponding oximes [7]. The oximes are subsequently converted into $\alpha$-hydroxynitriles by CYP71E7 [15], which are subsequently glucosylated by either UGT85K4 or UGT85K5 to produce linamarin and lotaustralin $[13,7,16]$. UDP-glucosyl transferase catalyzes the addition of glucose to acetone cyanohydrin in this last step of linamarin biosynthesis (Fig. 1a) [13,7]

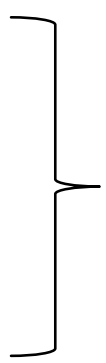

CYP79D1

CYP79D2
N-hydroxy valine<smiles>[AlH2]</smiles>

2-methyl propanol oxime<smiles>[AlH2]</smiles>

Acetone cyanohyd rin + UDP-glucose<smiles>CC(C)(C)C</smiles>

Linamarin

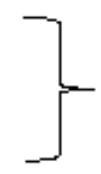

CYP71E7

UDP-glucosyl transferase

Figure 1 (a): Cyanogenic glucosides (CNglcs) biosynthetic pathway in cassava [7]
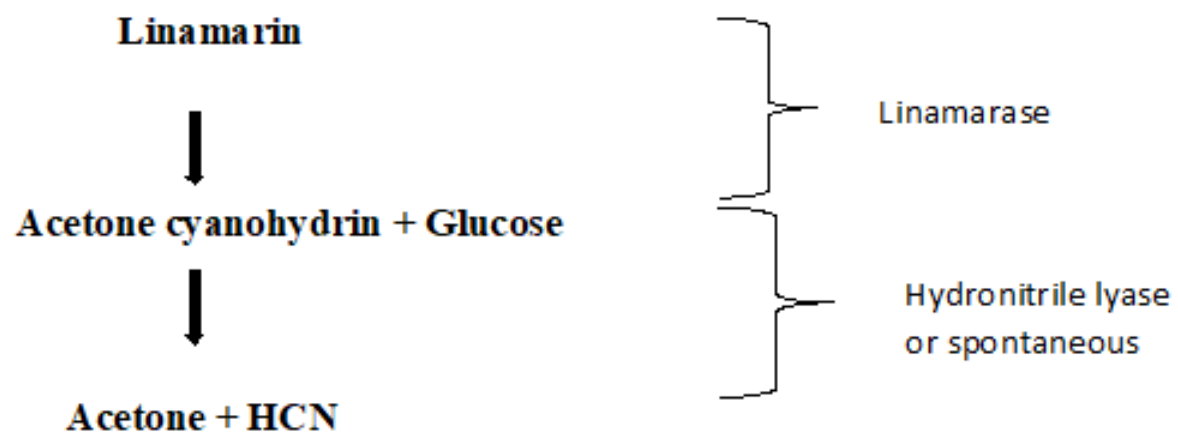

Figure 1 (b): Cyanogenesis in cassava [7]

In the last phase, the acetone cyanohydrins generated by CYP71E7 enzyme are unstable and are subsequently broken down into ketones and cyanide [15]. Oxime metabolizing enzymes are thought to be very efficient in order to avoid the release of free oximes, which are toxic to the plant $[17,18,19]$. Selmar [20] proposed that linustatin can be deglucosylated by sequential cleavage, in which the linustatin is converted to linamarin and stored in the vacuoles, or by simultaneous cleavage in which linustatin is converted in acetonecyanohydrin and produced free $\mathrm{HCN}$ at high $\mathrm{pH}$ and temperature, based on his findings in Hevea bransilensis. As a result, the plants must re-assimilate the $\mathrm{HCN}$ through 
detoxifying activities to avoid autotoxicity [21]. The presence of this detoxification pathway in cassava has been confirmed [21] and later research have revealed that $\beta$-cyanoalanine is the intermediate in the re-assimilation of cyanide to asparagine, which is mediated by $\beta$-cyanoalanine synthase and $\beta$-cyanoalanine hydrase [21].

The CNglcs synthesized from this pathway are stored in the vacuoles. When plant tissues are disrupted, they are exposed to the enzymatic activities of $\beta$-glucosidases (BGDs), which results in the hydrolysis and liberation of cyanohydrin aglycone, which then dissociates to liberate hydrogen cyanide (HCN) and a ketone either spontaneously or via catalysis by a leaf-specific enzyme, $\alpha$-hydroxynitrile lyase (HNL) [7]. This process is termed cyanogenesis and is regarded as the bioactivation pathway (Fig. 1b) [6].

\section{ADVANCES IN DEVELOPING CYANOGENIC GLUCOSIDES-FREE CASSAVA PLANTS}

The successful cloning of the two P450 genes encoding the enzymes that catalyze the initial steps in CNglcs biosynthesis, by Andersen et al. [14], provided the necessary molecular tools for further research in the development of acyanogenic cassava plants. Following this, Andersen et al. [14] proposed that acyanogenic cassava plants may be developed through blocking the synthesis of the two P450 enzymes, by knocking out the functions of CYP79D1/D2 genes using antisense or RNAi technologies. This concept of knocking out the functions of these genes spurred several research efforts in this field.

Siritunga and Sayre [22] developed cassava lines with reduced cyanide potential by the selective inhibition of CYPD1/D2 genes in the leaves or roots of cassava. The authors used an antisense technique to insert the $5^{\prime}$ ends (650bp) of the cytochrome $\mathrm{P} 450$ genes into cassava plants in a reverse orientation under the control of the leaf-specific Cab1 promoter. The transformed plants had 94\% reduction of linamarin content in the leaves. There was a reduction in root linamarin level ( $<1 \%$ of the wild-type plants) in transformed plants that had between 94 and $60 \%$ reduction in leaf linamarin content. Reductions in the transcript levels of these genes in the roots could not account for this, since transcript levels in transformed roots were similar to non-transformed plants [22]. A possible explanation could be due to a reduction in the level of linamarin transported from the leaves to roots. In that case, there is a level of leaf linamarin content required for linamarin to be translocated to the roots since transformed plants having a $40 \%$ of the non-mutant linamarin level had less than $1 \%$ of the non-mutant root linamarin level [22]. However, it was observed that there was no link between the reduction in $\mathrm{P} 450$ genes transcript levels and linamarin content in some transformed plants. This may reflect additional metabolic flux controls governing the rates of linamarin biosynthesis, translocation and storage [22].

It is worth noting that the silencing of similar genes in the roots alone had no effect on the linamarin levels of the cassava plants as reported by Siritunga and Sayre [22] and Siritunga and Sayre [7]. In a similar study by Siritunga and Sayre [7], the CYP79D1/D2 transcript levels were reduced to non-detectable levels using antisense expression of rootspecific patatin promoters. The patatin-CYP79D1/D2 transformed lines had unaltered root and leaf linamarin contents. However, transformed plants with antisense expression of Cab1-CYP79D1/D2 in the leaves had their leaf and root linamarin levels significantly reduced [7]. The result showed that in cassava lines of 3-4 months old, a reduction in leaf cyanide potential leads to a reduction of CNglcs in the roots. Unfortunately, the Cab1-CYP79D1/D2 antisense transformants developed by Siritunga and Sayre [7] failed to root when cultured in vitro in ammonia deficient media, and eventually withered after transfer to nitrogen deficient potting soil; however, patatin-CYP79D1/D2 antisense transformants thrived in ammonia deficient media, as well as potting soil.

Jørgensen et al. [8] also developed cassava lines with reduced CNglcs by targeting either or both genes encoding the first step enzymes using anti-sense constructs. The study produced significant reductions in cyanide level, as much as $80 \%$ in the leaves and $60 \%$ in the tubers. The researcher also employed an RNAi technique to generate cassava plants where reductions as high as more than $99 \%$ of the non-mutant type were obtained in the leaves. In some of the resulting transgenic plants, the linamarin contents in the tubers were reduced to $8 \%$ of the non-mutant amounts. Transgenic lines with less than $25 \%$ of the average cyanide contents had distinct morphological phenotypes when cultured in vitro, having long and slender stems, and either failed to produce normal roots or no roots at all, similar to what Siritunga and Sayre [7] observed; Although normal growth was restored with the supplementation of the growth media with nitrogen source [8]. In a similar study by Piero et al. [23], transgenic cassava lines were generated through Agrobacteriummediated transformation and an RNAi cassette containing cytochrome P450 genes (CYP79D1) was applied to down regulate the synthesis of $\mathrm{CNglcs}$ in the leaves. The analysis of cyanide levels in roots and leaves of both wild type and transgenic lines by the authors showed that cyanide levels were significantly reduced by three-fold in the transgenic lines.

The genes encoding the enzymes in the CNglcs catabolic pathway have also been targeted in cassava [22]. This pathway ensures the degradation of CNglcs into acetone and cyanide by the enzymes: linamarase and HNL [22]. These enzymes are reported to be present in the leaves in abundance, but less in the roots [24]. Linamarase and HNL have similar catalytic activities and expression levels in the leaves, which ensure proper cyanogenesis and food detoxification [24,1]. However, linamarase activity is lower in the roots with a low amount of HNL, which might explain the toxic levels of acetone cyanohydrin in improperly processed cassava roots [24]. The confirmation of the absence of linamarase mRNA in the roots of cassava by Santana et al. [25] suggesting that 
linamarase is not synthesized in the roots but rather, translocated to the roots via the laticifer system [1].

The over-expression of HNL in cassava tubers provides an effective alternative to the reduction of cyanogen in cassava tubers. This will hasten the breakdown of cyanogenic glucoside in the tubers during processing [7]. This led Siritunga and Sayre [7] to propose that the catabolic pathway may be exploited to develop lines that efficiently partition most of the linamarin present in the roots, towards amino acid biosynthesis, or by increasing the rate of its breakdown (cyanogenesis). As a result, the accumulation of linamarin in the tubers to toxic levels might be avoided.

Exploiting this mechanism, Siritunga and Sayre [7] regenerated cassava plants where HNL activity was overexpressed. It was observed that acetone cyanohydrin was converted to cyanide in 2 hours, following maceration under conditions favourable for their accumulation; significantly lowering the levels of acetone cyanohydrin. This is contrary to what was observed in non-mutant lines, where the quantity of acetone cyanohydrin remained constant under these conditions [7]. Importantly, the linamarin amounts in the transformed plants and wild-type plants were identical [7].

\section{Future Research Direction}

The objective of developing cassava plants completely free of CNglcs in the tubers has remained elusive in the past decades. $\mathrm{Du}$ et al. [26] found that cyanogenic glucosides are de novo synthesized in the cassava tubers. Therefore, it is difficult to predict the growth of acyanogenic cassava plants by downregulating its synthesis in the leaves alone. Despite the advances in developing lines with lower CNglcs levels, Jørgensen et al. [8] observed a significant reduction of CNglcs in the leaves, without a corresponding reduction in the roots; where mutants still retained the wild-type CNglcs levels in the roots. In addition, the inability of mutant lines regenerated by Siritunga and Sayre [7] and Jørgensen et al. [8] to develop properly, poses another challenge. This suggests more diversified roles played by CNgles in the plant, rather than just the traditional role as defense compounds. Therefore, further research in this field will have to follow a different path; one that conserves their roles, while constructively eliminates the compounds from edible tubers. There are two important pathways that should pique the curiosity of researchers, namely the endogenous turnover and the CNglcs transport channel.

An endogenous turnover of CNglcs has been reported in cassava [3]; although, this endogenous turnover pathway in cassava, unlike in other cyanogenic plants, is yet to be characterized. It is worth noting that in sorghum, dhurrin is produced and stored in developing seeds, but the mature seeds do not contain dhurrin since it is turned over during seed maturation $[27,3]$. In the same way, linamarin accumulates in mature seed of the rubber tree (Hevea brasiliensis), and it is converted to linustatin following germination; and then translocated into the cotyledon for turnover [27]. A similar turnover event was seen in bitter almonds [28]. It is obvious from these instances that the endogenous turnover of CNglcs reduces the amounts of $\mathrm{CNglcs}$ in various plant tissues.

According to Schmidt et al. [3], both transcript and protein levels regulate endogenous $\mathrm{CNglcs}$ turnover in cassava. The presence of this endogenous turnover pathway, is obvious in cassava where the intermediates are known, but none of the enzymes and genes involved have been identified [29,27]. The products of CNglcs endogenous turnover in cassava include linamarin amide, linamarin acid and linamarin anitrile [3]. These are found in trace amounts in cassava leaves, and their low levels may be due to their rapid incorporation into primary metabolism $[29,27,3]$. As a result, sufficient attention should be paid to this endogenous turnover pathway in cassava, which could lead to the identification of the genes and enzymes involved in the biosynthesis of these turnover products. It is expected that the upregulation of these genes would result in increased synthesis of these turnover products, which would then be incorporated into primary metabolism; thereby avoiding the accumulation of CNglcs in the tubers.

Another intriguing topic that has been met with less attention is the translocation of CNglcs from the leaves to cassava tubers. Unfortunately, the mechanisms of this transport channel are also unclear. This is not unexpected, since in studies of secondary metabolites for decades, full attention has always been given to the biosynthetic enzymes and genes, with little or no attention paid to the transport mechanisms [30]. It is a typical feature in plants to partition synthesis and storage of secondary metabolites in different organs; for example, tobacco synthesizes nicotine in the roots and transports via xylem to the shoot [19]; Coptis japonica synthesizes berberine in the roots and transports to starch-rich cells of the rhizome [31,32]; cassava synthesizes cyanoglucosides in the leaves and transports via the phloem to the roots $[8,15]$; and brassicas synthesize glucosinolates in maternal tissues and transports to the seeds [33,34]. As a result, defensive chemicals are often transported from the sites of synthesis to sink organs.

Several studies have proposed different mechanisms for this transport, such as simple diffusion, vesicle-mediated transport, and transporter-mediated membrane transport [30]. Four families of transporter proteins have been identified, including the ATP-binding cassette transporter (ABC) [35,36,37], nitrate-peptide transporter (NRT) [38,39], multidrug and toxic compound extrusion (MATE) [37,40]; and purine permease families (PUP) [41,30]. These transporters are large protein families and also play roles in the transport of primary metabolites and phytohormones. Majority of the ABC proteins consist of transmembrane domains (TMDs) and nucleotide binding domains (NBDs) [30]. (Shitan, 2016).

MATE proteins presumably function as proton antiporters and hormone signaling [37,40]. PUP transport substances which include purine ring-containing compounds like adenine, cytosine and cytokinins as well as pyridine ring-containing compounds like pyridoxine and pyridoxal [41]. Initially, 
NRTs were supposed to act as transporters for nitrates or peptides and function as proton symporters. However, reports recently revealed their involvement in the transport of defense compounds including glucosinolates, and phytohormones [38,39]. Glucosinolate transporters have been identified as Glucosinolate Transporter 1 (GTR1) and Glucosinloate Transporter 2 (GTR2) in Arabidopsis thaliana [38], belonging to the NRT family [30].

The identification of these important transport molecules of defense compounds in the Arabidopsis has given a longneeded model system for studying transport mechanisms of defense compounds in other plants [19]. These transporters are vital in the translocation of aliphatic and indole glucosinolate to Arabidopsis seeds, by loading the metabolites in the phloem of the leaves [38]. The proteins are also involved in the distribution of glucosinolates within the mature leaf, and between leaves and roots [14,42,43]. Some plants with altered expression of transport molecules showed significant changes in metabolite accumulation. For example, knocking out of glucosinolate transporters, GTR1 and GTR2, in Arabidopsis shows clearly reduced glucosinolate levels in seed, and down regulation of CrTPT2 enhances the synthesis of dimers of catharanthine and vindoline, which are precursors of the anti-cancer compound vinblastine $[38,44]$. This clearly demonstrates that metabolic engineering of these compounds are conceivable by interfering with the expression of transport molecules [45], and has the potential to lead to developing plants with reduced accumulation of toxic compounds in edible tissues.

\section{CONCLUSION}

The presence of cyanogenic glucosides in cassava has not only hampered its effective use as a food crop, but it has also slowed the progress in improving the nutritional values of cassava. Although processing of cassava reduces the cyanogen content of the crop to acceptable levels, which may be safe for consumption, the development of cyanogen-free cultivars is a priority to ensure a safer and more nutritious cassava, as well as to facilitate research in the areas of improvement for nutritional traits such as proteins, vitamins, minerals and other micronutrients.

The endogenous turnover pathway and CNglcs transport channel could be interesting areas for future research. CNglcs are thought to be transported in the form of linustatin due to its occurrence in other cyanogenic plants. However, the knowledge of the mechanisms of transport of defense compound has been hampered by the few transporters identified in model plants. As a result, the characterization of glucosinolate transporters in Arabidopsis opens an avenue to study the mechanisms and roles of transporters of defense compounds such as CNglcs in cassava. It is worth noting that defense compounds from different species show great similarities with regards to separation of biosynthesis and storage. This means that the analysis of glucosinolate transport pathways in Arabidopsis may be applied to other plant species and will, therefore, spur the study of defense compound transport as well as other specialized metabolites.

The last two decades have witnessed intense research in developing cassava plants with acceptable level of CNglcs, by suppressing the expression of the CYP79D1/D2 in the CNglcs biosynthetic pathway. These concerted efforts, as we have seen, are yet to produce a satisfactory outcome. Since research has revealed that $\mathrm{CNglcs}$ play diverse roles in plants, the idea of silencing these genes may not be the best approach because the elimination of CNglcs could affect other metabolic processes in the plant. As a result, alternative strategies must be developed to conserve the functions of these compounds in the plant, while removing the compounds from the edible tubers.

\section{CONFLICT OF INTERESTS}

The authors declare no conflict of interests.

\section{ACKNOWLEDGEMENT}

We wish to thank our colleagues in the Department of Plant Science and Biotechnology, University of Nigeria, Nsukka for their advice, support and constructive criticisms during the course of this work.

\section{REFERENCES}

[1] Siritunga D, Sayre R. Transgenic approaches for cyanogen reduction in cassava. Journal of AOAC International 2007; 90(5): 1450 - 1455 .

[2] Piero NM, Murugi NJ, Oduor OR, Ombori OR, Cheruiyot RC, Jalemba MA. Determination of cyanogenic compounds content in transgenic acyanogenic Kenyan cassava (Manihot esculenta Crantz) genotypes: Linking molecular analysis to biochemical analysis. Journal of Analytical \& Bioanalytical Techniques 2015; $6: 1-7$.

[3] Schmidt FB, Cho SK, Olsen CE, Yang SW, Møller BL, Jørgensen $\mathrm{K}$. Diurnal regulation of cyanogenic biosynthesis and endogenous turnover in cassava. Plant Direct 2018; 2(2):1 - 9.

[4] Andersen MD, Busk PK, Svendsen I, Møller BL. Cytochromes P450 from cassava (Manihot esculenta Crantz) catalyzing the first steps in the biosynthesis of the cyanogenic glucosides linamarin and lotaustralin. Journal of Biological Chemistry 2000; 275(3):1966 - 1975.

[5] Gleadow RM, Møller BL. Cyanogenic glycosides: synthesis, physiology and phenotypic plasticity. Annual Review of Plant Biology 2014; 65:155 - 185.

[6] Morant AV, Jørgensen K, Jørgensen C, Paquette SM, SánchezPérez R, Møller BL, Bak S. $\beta$-Glucosidases as detonators of plant chemical defense. Phytochemistry 2008; 69(9):1795 - 1813.

[7] Siritunga D, Sayre R. Engineering cyanogen synthesis and turnover in cassava (Manihot esculenta). Plant Molecular Biology 2004; 56(4):661 - 669 .

[8] Jørgensen K, Bak S, Busk PK, Sørensen C, Olsen CE, PuontiKaerlas J, Møller BL. Cassava plants with a depleted cyanogenic glucoside content in leaves and tubers. Distribution of cyanogenic glucosides, their site of synthesis and transport and blockage of the biosynthesis by RNA interference technology. Plant Physiology 2005; 139(1):363 - 374 .

[9] Møller BL. Functional diversifications of cyanogenic glucosides. Current Opinion in Plant Biology 2010; 13(3):338 - 347.

[10] Bak S, Kahn RA, Nielsen HL, Møller BL, Halkier BA. Cloning of three A-type cytochromes P450, CYP71E1, CYP98, and CYP99 from Sorghum bicolor (L.) Moench by a PCR approach and identification by expression in Escherichia coli of CYP71E1 as a multifunctional cytochrome $\mathrm{P} 450$ in the biosynthesis of the 
cyanogenic glucoside dhurrin. Plant Molecular Biology 1998; 36(3):393 - 405.

[11] Hansen KS, Kristensen C, Tattersall DB, Jones PR, Olsen CE, Bak $\mathrm{S}$, Møller BL. The in vitro substrate regiospecificity of recombinant UGT85B1, the cyanohydrin glucosyltransferase from Sorghum bicolor. Phytochemistry 2003; 64(1):143 - 151.

[12] Blomstedt CK, Møller BL, Neale AD, Gleadow RM, Bjarnholt N, Hamill JD, O'Donnell NH. Metabolic consequences of knocking out UGT85B1, the gene encoding the glucosyltransferase required for synthesis of dhurrin in Sorghum bicolor (L. Moench). Plant and Cell Physiology 2015; 57(2):373 - 386.

[13] Bak S, Olsen CE, Halkier BA, Møller BL. Transgenic tobacco and Arabidopsis plants expressing the two multifunctional sorghum cytochrome P450 enzymes, CYP79A1 and CYP71E1, are cyanogenic and accumulate metabolites derived from intermediates in dhurrin biosynthesis. Plant Physiology 2002; 123(4): 1437 - 1448 .

[14] Andersen TG, Nour-Eldin HH, Fuller VL, Olsen CE, Burow M, Halkier BA. Integration of biosynthesis and long-distance transport establish organ-specific glucosinolate profiles in vegetative Arabidopsis. Plant Cell 2013; 25(8):3133 - 3145.

[15] Jørgensen K, Morant AV, Morant M, Jensen NB, Olsen CE, Kannangara R, Motawia MS, Møller BL, Bak S. Biosynthesis of the cyanogenic glucosides linamarin and lotaustralin in cassava: isolation, biochemical characterization and expression pattern of CYP71E7, the oxime-metabolizing cytochrome P450 enzyme. Plant Physiology 2011; 155(1):282 - 292.

[16] Kannangara R, Motawia MS, Hansen NKK, Paquette SM, Olsen CE, Møller BL, Jørgensen K. Characterization and expression profile of two UDP-glucosyltransferases, UGT85K4 and UGT85K5, catalyzing the last step in cyanogenic glucoside biosynthesis in cassava. The Plant Journal 2011; 68:287 - 301.

[17] Grootwassink JWD, Balsevich JJ, Kolenovsky AD. Formation of sulfatoglucosides from exogenous aldoximes in plant cell cultures and organs. Plant Science 1990; 66(1):11 - 20.

[18] Bak S, Olsen CE, Petersen BL, Møller BL, Halkier BA. Metabolic engineering of P-hydroxybenzyl glucosinolate in Arabidopsis by expression of the cyanogenic CYP79A1 from Sorghum bicolor. The Plant Journal 1999; 20(6):663 - 671.

[19] Jørgensen ME, Nour-Eldin HH, Halkier BA. Transport of defense compounds from source to sink: lessons learned from glucosinolates. Trends in Plant Science 2015; 20(8):508 - 514.

[20] Selmar D. Translocation of cyanogenic glucosides in cassava. Acta Horticulture 1994; 375:61 - 67

[21] Echeverry-Solarte M, Siritunga D, Figueroa A, González E, Ocasio-Ramirez V. Expression profiling of genes associated with cyanogenesis in three cassava cultivars containing varying levels of toxic cyanogens. American Journal of Plant Sciences 2013; 4:1533 -1545.

[22] Siritunga D, Sayre RT. Generation of cyanogen-free transgenic cassava. Planta 2003; $217(3): 367$ - 373

[23] Piero NM, Murugi NJ, Okoth OR, Ombori OR, Jalemba MA, Chelule CR. RNAi-mediated down regulation of cyano-glycoside biosynthesis in Kenyan cassava (Manihot esculenta Crantz) genotypes. Journal of Plant Biochemistry and Physiology 2014; 3(3):148 - 153.

[24] White WLB, Arias-Garzon DI, McMahon JM, Sayre RT. Cyanogenesis in cassava: the role of hydroxynitrile lyase in root cyanide production. Plant Physiology 1998; 116(4):1219 - 1225.

[25] Santana MA, Vasquez V, Matehus J, Aldao RR. Linamarase expression in cassava cultivars with roots of low- and high-cyanide content. Plant Physiology 2002; 129(4):1686 - 1694.

[26] Du L, Bokanga M, Møller BL, Halkier BA. The biosynthesis of cyanogenic glucosides in roots of cassava. Phytochemistry 1995; 39:323 - 326.

[27] Nielsen LJ, Stuart P, Pičmanová M, Rasmussen S, Olsen CE, Harholt J, Møller BL, Bjarnholt N. Dhurrin metabolism in the developing grain of Sorghum bicolor (L.) Moench investigated by metabolite profiling and novel clustering analyses of time-resolved transcriptomic data. BioMed Central Genomics 2016; 17:1 - 24.
[28] Sánchez-Pérez R, Jørgensen K, Olsen CE, Dicenta F, Møller BL. Bitterness in almonds. Plant Physiology 2008; 146(3):1040 1052.

[29] Pičmanová M, Neilson EH, Motawia MS, Olsen CE, Agerbirk N, Gray CJ, Flitsch S, Meier S, Silvestro D, Jørgensen K, SánchezPérez R, Møller BL, Bjarnholt N. A recycling pathway for cyanogenic glycosides evidenced by the comparative metabolic profiling in three cyanogenic plant species. Biochemical Journal 2015; 469(3):375 - 389 .

[30] Shitan N. Secondary metabolites in plants: transport and selftolerance mechanisms. Bioscience, Biotechnology and Biochemistry 2016; 80(7):1283 - 1293.

[31] Shitan N, Bazin I, Dan K, Obata K, Kigawa K, Ueda K, Sato F, Forestier C, Yazaki K. Involvement of CjMDR1, a plant multidrug-resistance-type ATP-binding cassette protein, in alkaloid transport in Coptis japonica. National Academy of Sciences of the United States of America Conference Proceedings 2003; 100(2): $751-756$.

[32] Shitan N, Dalmas F, Dan K, Kato N, Ueda K, Sato F, Forestier C, Yazaki K. 2013. Characterization of Coptis japonica CjABCB2, an ATP-binding cassette protein involved in alkaloid transport. Phytochemistry 2013; 91:109-116.

[33] Brudenell AJP, Griffiths H, Rossiter JT, Baker DA. The phloem mobility of glucosinolates. Journal of Experimental Botany 1999; 50:745- 756

[34] Chen S, Petersen BL, Olsen CE, Schulz A, Halkier BA. Longdistance phloem transport of glucosinolates in Arabidopsis. Plant Physiology 2001; 127(1):194 - 201.

[35] Verrier PJ, Bird D, Burla B, Dassa E, Forestier C, Geisler M, Klein M, Kolukisaoglu U, Lee Y, Martinoia E, Murphy A, Rea PA, Samuels L, Schulz B, Spalding EJ, Yazaki K, Theodoulou FL. Plant ABC proteins: a unified nomenclature and updated inventory. Trends in Plant Science 2008; 13:151 - 159.

[36] Yazaki K, Shitan N, Sugiyama A, Takanashi K. Cell and molecular biology of ATP-binding cassette proteins in plants. The International Review of Cell and Molecular Biology 2009; 276:263 - 299 .

[37] Shoji T. ATP-binding cassette and multidrug and toxic compound extrusion transporters in plants: a common theme among diverse detoxification mechanisms. The International Review of Cell and Molecular Biology 2014; 309:303 - 346.

[38] Nour-Eldin HH, Andersen TG, Burow M, Madsen SR, Jørgensen ME, Olsen CE, Dreyer I, Hedrich R, Geiger D, Halkier BA. NRT/PTR transporters are essential for translocation of glucosinolate defence compounds to seeds. Nature 2012; 488(7412):531 - 534.

[39] Nour-Eldin HH, Halkier BA. The emerging field of transport engineering of plant specialized metabolites. Current Opinion in Biotechnology 2013; 24(2):263 - 270.

[40] Takanashi K, Shitan N, Yazaki K. The multidrug and toxic compound extrusion (MATE) family in plants. Plant Biotechnology 2014; 31(5):417 - 430.

[41] Jelesko JG. An expanding role for purine uptake permease-like transporters in plant secondary metabolism. Frontiers in Plant Science 2012; 3:1 - 5 .

[42] Andersen TG, Halkier BA. Upon bolting the GTR1 and GTR2 transporters mediate transport of glucosinolates to the inflorescence rather than roots. Plant Signaling and Behavior 2014; 9(1):e27740.

[43] Madsen SR, Olsen CE, Nour-Eldin HH, Halkier BA. Elucidating the role of transport processes in leaf glucosinolate distribution. Plant Physiology 2014; 166(3):1450 - 1462.

[44] Yu F, De Luca V. ATP-binding cassette transporter controls leaf surface secretion of anticancer drug components in Catharanthus roseus. National Academy of Sciences of the United States of America Conference Proceedings 2013; 110(39): 15830 - 15835.

[45] Schroeder JI, Delhaize E, Frommer WB, Guerinot ML, Harrison MJ, Herrer-Estrella L, Horie T, Kochian LV, Munns R, Nishizawa NK, Tsay YF, Sanders D. Using membrane transporters to improve crops for sustainable food production. Nature 2013; 497(7447):60 - 66. 